\title{
Models for Integrated Pest Management with Chemicals in Atmospheric Surface Layers
}

\author{
${ }^{1}$ Bisignanesi, V. and ${ }^{2} M$. S. Borgas \\ ${ }^{1}$ Monash University, School of Mathematical Sciences, ${ }^{2}$ CSIRO Marine and Atmospheric Research, \\ E-Mail: valerio.bisignanesi@csiro.au
}

Keywords: Integrated pest management, pheromones, plume tracking; surface layer meteorology.

\section{EXTENDED ABSTRACT}

Surface layer landscapes are described by spatial distributions of canopy type and roughness, surface slopes and topography. Agricultural landscapes also include distributions of pests and pest management systems. Insect populations together with pheromone traps or dispensers constitute a system that depends on the airborne chemical 'landscape' shaped by the emissions and wind dispersal. Effective operation of such complex systems needs integrative tools comprised of Geographic Information System platforms coupled with dynamic component modelling.

We describe a linked modelling system which reduces uncertainty in monitoring for pest management. The spatial information from surface-layer landscapes and winds is integrated over multiple length and time scales. Larger scale meteorological flows $(\sim 5 \mathrm{~km})$ disperse pheromones from sources embedded in the surface layer canopy. Smaller scale plume properties $(\sim 100 \mathrm{~m})$, are downscaled even further to internal plume-fluctuation properties within the plumes $(\sim 1 \mathrm{~cm})$. Finally, the distributed plume properties are coupled with insect tracking models for pest distribution prediction.

The nature of these linked models is illustrated in the flow chart of Fig. 1:

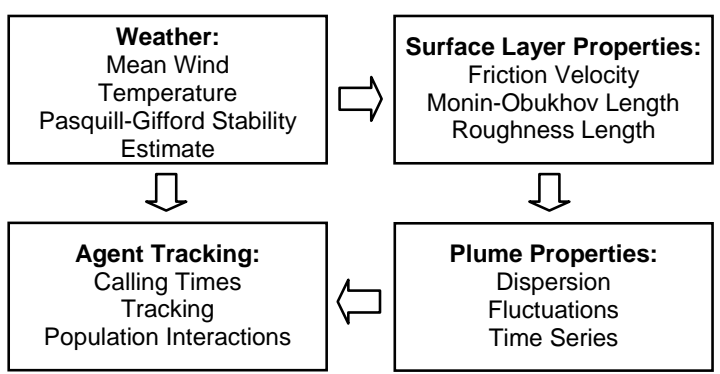

Figure 1. Schematic design of linked models for agent tracking.
The model hierarchy is simple, with the key being the parameters that are passed from one model level to the next.

Meteorological models, like the commercially available TAPM system, can model the weather, i.e. the broad scale mean flow over the terrain and determine the local stability over each surface type in the landscape. The complex chemical landscape within the wind fields in the surface layer can then be predicted with Lagrangian particle models of the dispersion of pheromone plumes from canopy releases. Such sources can be from either attracting females or synthetic disruption systems like traps. The dispersion models employed can effectively use outputs from the weather models or routine weather measurements.

The internal structure within the plumes, generated by surface layer turbulence, is also explicitly linked with surface layer characteristics of the fine scale turbulence but at even smaller scales because of the tiny dimensions of the source. We adapt classic Monin-Obukhov similarity theory (Stull 1988) for modelling plume fluctuations and develop instantaneous random concentration field properties based on inertial range scaling of turbulent processes.

The final component is an agent-based insect tracking model that couples directly to the local instantaneous chemical landscape. The intelligent agent makes navigation decisions based on chemical signals to home onto the target source.

The coupled system provides an insect mating prediction that accounts for trap catch counts and the influence of weather and cropping systems on these processes. Studies with this model will ultimately allow for a more accurate inference from trap monitoring and for the better management of pests. 


\section{INTRODUCTION}

Insect pests cause substantial damage to agriculture worldwide by attacking the crops in fields and in storage. For example, helicoverpa spp. cause an estimated $\$ 225 \mathrm{M}$ damage to Australian agriculture per annum (QLD DPI).

Traditional management strategies rely on the use of pesticides. This approach has proved widely effective for controlling pests but has costs due to:

- Expensive chemicals and applications;

- Waste in poor meteorology; and

- Emergence of pesticide resistance in insect pests requiring new formulations.

Environmental costs include:

- Hazardous exposure to poisonous chemicals;

- Possible off-target and downwind drift of the insecticide;

- Pollution of underground water bodies and soils; and

- Loss of beneficial insect biodiversity.

In general, the use of pesticides needs to be minimized for sustainable agriculture.

\subsection{Integrated Pest Management}

Starting in the 1970s alternative forms of pest management have emerged. The term Integrated Pest Management (IPM) refers to the adoption of a linked set of strategies, ranging from monitoring and the limited strategic use of pesticides, to the adoption of modified crops with refuges for beneficial insects. Included is the interaction with natural pheromone attractants, either for monitoring with traps, or for mating disruption (Cardé and Minks 1995).

Unfortunately, the adoption of IPM programs has been patchy and in most cases limited to an optimization of the use of pesticides (Fitt et al. 2004). This is partly due to the complexity of the interactions, environmental uncertainty and poor knowledge about the state of the system.

The constant monitoring of crop fields is critical to the success of an IPM strategy. One of the key indicators is the pest number density. The critical number of insects per area which "can eat or destroy more dollars' worth of grain than the cost of spraying" is important (QLD DPI). This critical number is also referred to as the economic threshold above which a control action is economically justified and desirable. Being able to predict this critical threshold, or simply be alerted quickly when it has been reached, is an important part of managing the system.

Estimating pest number density is a non trivial task that is often performed indirectly by assessing damage in the field or by using traps for monitoring the number of individuals. Traps baited with an attractive sex-pheromone mixture are extensively employed for this purpose. However, reliably linking pheromone trap counts to pest number density is a challenge.

Many insect pests are highly mobile species capable of both inter-field and migratory travel. In order to successfully track mating partners, insects have developed remarkable abilities to detect vanishingly small concentrations of pheromones. Because of the high insect mobility, the key to interpreting trap catches is the evaluation of the area of attraction (footprint) of the trap. This footprint is strongly dependent on the searching strategy used by the insect to locate the source of the pheromone plume. It is also controlled by the turbulent dispersion of the pheromone in the air (Murlis et al. 1992).

The important factors for plume-tracking by insects, and models to characterize trap footprints, are the topics of this paper. The key elements are the interaction of:

- An intelligent agent (the insect);

- Random environmental stimulus (the pheromone signal); and the

- Influence and control of the weather.

Each element can be modelled and, importantly, linked with the others to reduce the large uncertainties in the trap footprint problem.

\subsection{Physical Models}

The nature of the chemical signature (filamentary structure) is determined by the turbulent mixing process that takes place in the natural environment. Insects have evolved to exploit these small scale structures to guide their way to their mating partners (Murlis et al. 1992).

Turbulent mixing in the surface layer of the atmosphere is strongly affected by local meteorological conditions and surface properties. For a particular example, moths tend to track at night, thus the role of atmospheric stability on pheromone dispersion is crucial. Meteorological conditions are also important because of their direct influence on insect behaviour. For example, temperature regulates both the onset of female calling and male responsiveness (Bell et al. 1995). 
The searching algorithm used by an insect "agent", has been observed to consist of a combination of upwind flight if the pheromone is detected, and cross-stream zigzag counterturns in clean air. The upwind motion is used to get closer to the target when the pheromone is sensed, while the casting flight is adopted when no signal is sensed in order to maximize the chances of filament interception. This is a simplified description of the searcher behaviour (Bell et al. 1995), nevertheless, it represents a successful strategy to robustly guide the insect to the source of the pheromone.

While the detection and tracking behaviour of insects have been extensively studied by entomologists, pheromone dispersion is still poorly understood and represents a significant source of uncertainty (Murlis et al. 1992). While there have been attempts in the literature to model the finescale structure of the concentration field (Farrell et al. 2002), what is still missing is a general tool for predicting pheromone plume properties in the natural environment.

\subsection{Outline for a Linked Model System}

The schematic shown in Fig. 1 illustrates the proposed integrated system. More detail is outlined below. The agent tracking process is affected by the meteorological conditions (e.g. temperature) and by properties of the chemical signal and is discussed briefly in $\$ 2$. The characterization of the broad scale flow and its influence on the surface layer properties are discussed in $\$ 3$. $\$ 4$ describes how the dispersion in the surface layer affects the turbulent mixing and the filamentary structure of the chemical signal. Some simple results from the integrated model are presented in $\S 5$ and the conclusions in $\S 6$. The principal results presented here are number density estimates for a simple trap in light-wind neutral conditions and in stable conditions. We solve for insect tracking in a large domain downwind of the trap for a calling/tracking period of two hours. The key results for inferences from trap catches as relevant for pest management are discussed.

\section{PLUME TRACKING MODELLING}

Plume tracking refers to the process of an insect following an attractant chemical plume. This basic process controls the pest dynamics in complex interacting populations in spatially varying crops and surface vegetation. Trapping pests to estimate numbers often involves a baited scent trap to attract male pests from downwind. This is characterized by a footprint of attraction which depends on:
- The dispersion by wind and turbulence downwind of the trap;

- The likelihood of encountering females before reaching the trap;

- The effectiveness of tracking in the given conditions; as well as

- The number of individuals on average in the system.

The system is complex and not conveniently modelled by traditional systems like diffusion models or simple random walks. However, the use of agent based systems (Łomnicki 1992) is a natural framework and permits a direct link between the modelled catch numbers and number density. Simulation also allows for statistical uncertainty of the system to be determined.

\subsection{Individual Agent Tracking}

An individual insect agent tracking a single plume (either from a calling female or a baited trap) is itself a complex process. This is mainly due to the variability of the plume in the atmosphere as controlled by winds. However, insects have adapted to this reality. Their behaviour can be represented by a rule-based agent which is assumed to make flying decisions based on the detected pheromone concentration. The rules are:

- Fly upwind above detection thresholds of the chemical signal (called surging); and

- $\quad$ Fly cross wind (back and forth) when not detecting a chemical signal (called casting).

The parameters for the agents (flying speeds, meteorological conditions for calling and tracking, detection thresholds for various pheromone signals) are reasonably well known and some are listed below.

Even in a simple two agent system (caller and tracker) the process is a random walk because the signal detection is an instantaneous sample of a random (stochastic) and highly variable chemical signal due to atmospheric turbulence. It is unlikely that insects have processing power and memory to form averages and complex spatial-pattern recognition for plume properties. Indeed they have no need for sophistication because the casting and surging tracking rules almost always guarantee success given sufficient time.

Insects are capable of remarkable tracking feats following minute calling emissions, even over 
distances of several kilometres. Some of the typical characteristics of insect agent tracking are:

- Cycle time for decisions: $150-500 \mathrm{~ms}$

- Flying speed:

$1 \mathrm{~m} / \mathrm{s}$

- Detection thresholds: $10^{3} \mathrm{~mol} . / \mathrm{cm}^{3}$ (integration time: $1 \mathrm{~s}$ )

- Calling period:

2 hours

- Calling emissions: molecules/s)

- Calling temperature:

$1 \mathrm{ng} / \mathrm{min}\left(10^{10}\right.$

20-25 deg. C

\subsection{Multiple Agents}

In a multi-agent system (including the crop manager with his or her trap), the basic tracking rules for a tracking agent are the same, but the chemical field now consists of a multitude of plumes dispersing and mixing simultaneously. The tracking can randomly follow any plume, simply biased by the probability of the detection being from one plume or another. From the point of view of the tracker, or an individual caller, this is really no more complex than the basic two agent system.

\subsection{Chemical Signal Structure}

The key feature of a model system is the atmospheric variability of the chemical signals. In the most complex situation we have:

- Many stationary point sources emitting downwind;

- Highly variable signals in time and space;

- $\quad$ Strong dependence on meteorology and surface layer processes; and

- Complex modelling of the basic physical state of the system.

The basic chemical state is familiar from classical boundary layer dispersion for air quality assessment, particularly odour problems (Borgas 2000). Here the emphasis is on the near surface conditions and smaller scales of interest, both for source configurations and sampling scales.

Despite the complexity there is a clear strategy for constructing an integrated model linking trap catch with the key system variables:

- The number density of pests;

- The variability due to meteorology;

- The statistical variability of the inherent stochastic process; and
- $\quad$ Alternative agent strategies (lure and kill, mating disruption, smart tracking).

Calling and tracking are strongly controlled by meteorology. Calling commences at dusk with slackening winds and turbulence. Sufficient meteorological modelling to reflect the influence of stable surface layer dispersion is needed in the model. This ultimately reduces the overall uncertainty in the pest-catch estimates by allowing systematic analysis of the role of weather variability in the modelled pest response.

\section{SURFACE LAYER MODELLING}

The generic surface layer problem we consider is the calling emission at a height $H$ in the vegetation canopy. We assume sparse vegetation of some relatively homogeneous crop, at heights of one to two metres.

The calling plume is advected by a mean wind, imposed by broader scale weather patterns. However, most of the processes in the near surface layer that control the calling plume and the insect tracking can be understood in terms of local variables. This framework, known as MoninObukhov similarity theory (Stull 1988) is a powerful representation of the surface layer activity and here we utilise extensions for plume modelling for tracking purposes.

\subsection{Integrating Weather Information}

Monin-Obukhov similarity theory is a basic component of weather-prediction model parameterization. Conversely, weather prediction or assessment can be used to infer similarity parameters (NSW EPA 2001). The link is provided by relating the mean wind properties and classical Pasquill-Gifford stability estimates to $u_{*}$ (friction velocity) and $L$ (Monin-Obukhov length), and then further linking fine scale turbulent fluctuations of plumes to models based on parameterization by $u_{*}$ and $L$. This allows for relatively widespread application, as practiced in air pollution assessment (NSW EPA 2001) models, including relevant parameters for specific crops, weather conditions, pest types and trap distributions, which can be modelled as an integrated system. More certainty can be obtained by directly assimilating local micro-meteorological measurements. Increasingly crop management will integrate such measurements to manage fluxes of carbon, nitrogen and water with additional benefits for pest management applications. 


\subsection{Monin-Obukhov Similarity Theory}

The basic theory for the dynamics of the model includes expressions for the:

- $\quad$ Mean wind, $\bar{U}(z)$, as a function of height;

- Turbulent fluctuations as a function of stability; and

- The dissipation rate of turbulent kinetic energy as a function of height.

The properties depend on two key parameters, the friction velocity $u_{*}$ (typical of fluctuating winds near the surface) and the Monin-Obukhov length $L$ which reflects a length scale over which stability effects control vertical motions (it is smaller in strong stability when vertical motions are confined closer to the surface). Stable layers are the focus of modelling for plume tracking because calling is often initiated at evening when cooling layers of air near the surface become denser and less prone to vertical motion.

However, even in stable layers the horizontal motion of the wind induces frictional turbulent motions requiring an extra roughness length parameter, $z_{0}$, to be included in the representation. The combination of the two parameters, $u_{*}$ and $L$, describe much of the behaviour in well established functional forms for the dynamic variables. For given weather conditions, either measured at a micro-meteorological station or inferred from larger scale weather, the state of the surface layer can be prescribed. As an example, the key dynamic variable for linking the model hierarchies is the dissipation rate of turbulent kinetic energy, $\bar{\varepsilon}(z)$, which describes the local activity of the turbulence. The empirical form is:

$$
\bar{\varepsilon}=\left\{\begin{array}{l}
\frac{u_{*}^{3}}{\kappa z}\left(1.4+4.3 \frac{z}{L}\right) \quad z \geq z_{0} \\
\frac{u_{*}^{3}}{\kappa z_{0}}\left(1.4+4.3 \frac{z_{0}}{L}\right) z<z_{0}
\end{array}\right.
$$

which includes both frictional and stability effects (Han et al. 2000). The von Karman constant $\kappa$ is 0.4 .

\section{3. $\quad$ Plume Properties}

A surface-layer plume also satisfies similarity properties of its own. For many purposes, in air quality applications for example, the plume concentration is modelled only as an ensemble mean (an average over a large number of independent realizations of an emitted plume). This is not adequate for plume tracking which requires a stochastic representation of the instantaneous concentrations at the tracking agent receptor. Nevertheless, the mean concentration, $\bar{C}$, is a crucial aspect of the plume concentrations, and it is straightforward to model.

Plume tracking can be integrated into the MoninObukhov framework, over scales of several hundreds of metres horizontally, and within several metres of the surface. The mean concentration component of the model has the following features:

- Gaussian plume profiles;

- Dispersion parameters based on similarity parameters; and

- $\quad$ Stable to neutral layer mixing.

We also choose our parameterizations based on Lagrangian concepts of mixing (Sawford 2001), with a simple exponential auto-correlation of velocity for the lateral dispersion (across the plume horizontally) and a vertical auto-correlation with a negative loop for large lags reflecting stability effects, chosen to restrict the vertical mixing.

\section{CONCENTRATION FLUCTUATIONS}

Modelling fluctuating plume concentrations depends on (Borgas 2000):

- Plume fluctuations from Lagrangian pair modelling;

- An assumed probability density function for instantaneous concentrations;

- Clean air intermittency fractions within the plume; and

- Time series of jumps for spatial and temporal variability.

All depend on fine scale turbulence structure with meteorological control through the dependence of the pair dispersion on Monin-Obukhov scaling. This is achieved by modelling the pair separation probability density function, $P(r, t)$, for all pairs at given downwind plume cross sections in the emitted chemical plume (Borgas et al. 2005; Borgas and Yeung 2004). This is most simply modelled as a diffusion process where the diffusivity, for some number $\alpha$, is given by the mean-concentration weighted average:

$$
D=\alpha r^{4 / 3} \int_{-\infty}^{\infty} \int_{0}^{\infty} \bar{\varepsilon}^{1 / 3} \bar{C} d z d y=\alpha r^{4 / 3} \bar{\varepsilon}_{C}^{1 / 3}
$$

which incorporates the link to Monin-Obukhov similarity parameters through equation (1) and the 
mean concentration dependence. The diffusivity $D$ is a function of separation $r$ (so the pair separation is a non-Gaussian process). It is also time dependent which reflects the changing vertical sampling by the plume downwind.

The solution of the diffusion process gives the spatial correlations and intensity of the chemical concentration fluctuations. For zero separation the concentration variance is given by:

$$
\overline{C^{2}}(x)=\left.\overline{C^{2}}(0) P(r, t)\right|_{r=0} /\left.P(r, 0)\right|_{r=0} \quad x=\bar{U} t
$$

A broad variety of source distributions can be modelled with this framework. The fluctuation intensity, $\sigma_{C}^{2}=\overline{C^{2}} / \bar{C}^{2}-1$, reflects internal plume variability. Large fluctuations mean that insects can detect threshold concentrations even when the mean decays to undetectable levels. For the small sources considered here it is easy to dilute the mean plume by seven orders of magnitude as suggested by the parameters $\$ 2.1$. The spatial pair correlation of concentrations in the plume describes both the intensity of plume fluctuations and the spatial scale over which correlations occur thus determining the persistence of fluctuations.

\subsection{Concentration Jumps}

As an agent tracks a plume it senses concentrations that persist for very short times because of turbulent mixing. The change occurs as a sharp jump (Borgas et al. 2004). Even if the agent remains stationary at a fixed point, the chemical field sensed is punctuated by jumps along a time axis. The correlation in time of the jumps, and the concentration level in the span between jumps, can be simply fixed with the modelled statistics of the instantaneous plume concentrations and by using the two-time correlation in the plume, which is proportional to $\left.P(r, t)\right|_{r=\bar{U} \tau}$ for time lag $\tau$.

Taken together we can prescribe the character of instantaneous concentrations and the stochastic nature of how they change in time from the perspective of a tracking agent. This behaviour is directly linked to the meteorological parameters and the controlling influence of the weather. Thus we have a model capable of producing tracking responses sensitive to changing weather parameters and able to assess the relative tracking processes in varying weather conditions.

\section{PLUME TRACKING RESULTS}

The integrated system can now be solved for tracking behaviour. These will clearly be stochastic random walks tracking from downwind to the calling source. For example, starting an agent 100 metres downwind just beyond the threshold detection at mean concentration levels gives a typical path as shown in the schematic diagram (Fig. 2).

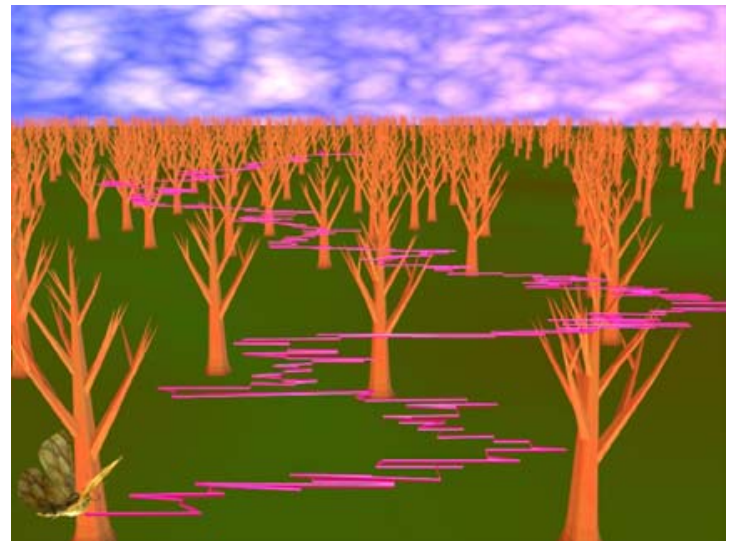

Figure 2. Snapshot from an example animation.

The random path (shown in pink) in Fig. 2 casts side to side, but systematically progresses upwind (wind comes out of the page) to a calling female.

Animations of plume tracking are a useful way to present the model output and the figure above is a sample shot of plume tracking animations generated by our system.

In general, for pest management we require statistics of tracking times. We use the agent model embedded in the surface layer landscape to evolve an ensemble of trajectories, i.e. a large number of independent tracking events are modelled. Neutral and stable conditions $(L=50 \mathrm{~m})$, are examined. Other conditions are fixed to be the same: $u_{*}=0.2 \mathrm{~m} / \mathrm{s}, z_{0}=10 \mathrm{~cm}$. The starting positions uniformly cover space downwind of the source, with unit number density (one agent per square metre). The trap source is strong with the mean concentration above the detection threshold up to $50 \mathrm{~m}$ downwind. Tracking is allowed for a maximum of two hours and the agent parameters are as listed in $\$ 2.1$. We also choose larger lateral diffusion for the neutral case.

\subsection{Stability Effects for Tracking}

For general number density, $n$, the two cases modelled here give trap-catch numbers $N_{\text {trap }} \sim 2400 n$, for the neutral case, which is $33 \%$ larger than the stable case $N_{\text {trap }} \sim 1800 n$. If the traps are used as indicators, then a single detection, on average, is associated with a number density of about one insect in each area of 2000 square metres. However, this number will depend on the threshold limit of detection for a particular trap emission, which would require more information.

The distributions we show below are the tracking time histograms, conditional on successful 
tracking. These are biased to large tracking times because the source is at one edge of the domain.

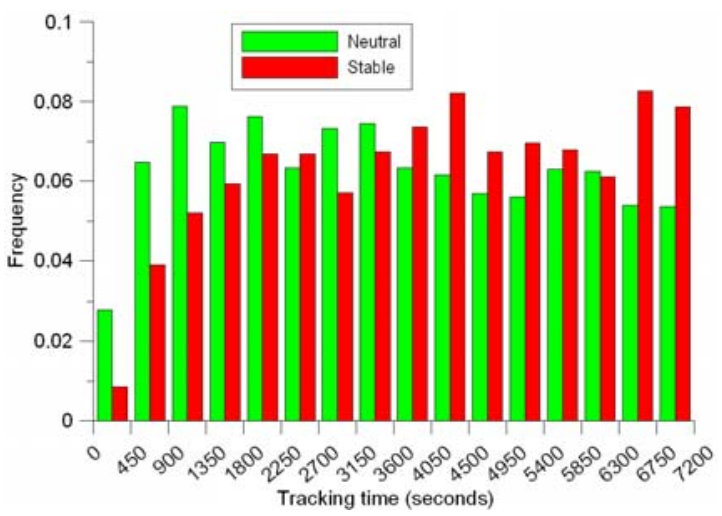

Figure 3. Tracking times frequency.

The results in Fig. 3 show a greater proportion of stable-layer paths tracked for longer times, but the enhanced plume spreading (in this example) in the neutral case mean that more tracking occurs from offset lateral agents.

\section{CONCLUSIONS}

Plume tracking models coupled with weather information (or models), dispersion and turbulence models, allow new estimates to be made for system inference in Integrated Pest Management. In particular, we have determined for the first time trap footprint estimates, and are developing a framework to calculate the link between trap catch and population number density in realistic meteorological conditions. The results show significant dependence on the meteorological stability parameters, and so these new models offer a way to reduce the current large uncertainty from monitoring inference from trap counts.

\section{BIBLIOGRAPHY}

Bell, W. J., L. R. Kipp and R. D. Collins (1995). The Role of Chemio-orientation in Search Behavior. In Chemical Ecology of Insects 2 (eds Cardé R. T., Bell W. J.), Chapman \& Hall, pp. 105-52, New York.

Borgas, M. S., B. L. Sawford, S. Xu, D. A. Donzis and P. K. Yeung (2004). High Schmidt Number Scalar Fields in Stationary Isotropic Turbulence. Physics of Fluids, 16, 3888-3899.

Borgas, M. S. and P. K. Yeung (2004). Relative dispersion in isotropic turbulence. Part 2. A new stochastic model with Reynolds-number dependence. Journal of Fluid Mechanics, 503, 125-160.

Borgas, M. S. (2000). The mathematics of whiffs and pongs [electronic publication]. In Enviro 2000: towards sustainability: four environmental conferences in one [CD-ROM]. 9-13 Apr 2000, Sydney, Australia.

Borgas, M. S., V. Bisignanesi, R. Gailis and S. T. Siems (2005). Concentration fluctuations in surface layers. In preparation for Bound. Layer Meteorol.

Cardé, R. T. and A. K. Minks (1995). Control of moth pests by mating disruption: successes and constraints. Annual Reviews of Entomology, 40, 559-85.

Farrell, J. A., J. Murlis, X. Long, W. Li and R. T. Cardé (2002). Filament-Based Atmospheric Dispersion Model to Achieve Short TimeScale Structure of Odor Plumes. Environmental Fluid Mechanics, 2, 143-169.

Fitt, G., L. Wilson, R. Mensah and J. Daly (2004). Advances with Integrated Pest Management as a component of sustainable agriculture: the case of the Australian cotton industry. In New directions for a diverse planet, Proceedings of the $4^{\text {th }}$ International Crop Science Congress 26 Sep - 1 Oct 2004, Brisbane, Australia.

Han, J., S. P. Arya, S. Shen and Y. Lin (2000). An Estimation of Turbulent Kinetic Energy and Energy Dissipation Rate Based on Atmospheric Boundary Layer Similarity Theory, Rep No CR-2000-210298. NASA Langley Research Center, Hampton VA, USA.

Łomnicki A. (1992). Population Ecology from the Individual Perspective. In Individual-based Models and Approaches in Ecology (eds D. L. DeAngelis and L. J. Gross), Chapman \& Hall, pp. 3-17, New York.

Murlis, J., J. S. Elkinton and R. T. Cardé (1992). Odor plumes and how insects use them. Annual Reviews of Entomology, 37, 505-32.

NSW Environment Protection Authority (2001). Approved methods and guidance for the modelling and assessment of air pollutants in New South Wales. p.50 Rep No EPA 2001/57 www.epa.nsw.gov.au/air/amgmaap.

Queensland Department of Primary Industries and Fisheries. Insect management in Queensland field crops. http://www.dpi.qld.gov.au/fieldcrops/9436.ht $\underline{\mathrm{ml}}$ retrieved on $18 / 08 / 05$.

Sawford, B. L. (2001). Turbulent Relative Dispersion. Annual Reviews of Fluid Mechanics. 33, 289-317.

Stull, R. B. (1988). An Introduction to Boundary Layer Meteorology. Kluwer Academic Publishers, 653 pp., Dordrecht. 